\title{
Uncertainty Quantification of Exponential Synchronization for a Novel Class of Complex Dynamical Networks with Hybrid TVD Using PIPC
}

\author{
Lin Pan ${ }^{1}$, Holger Voos ${ }^{1}$, Yumei $\mathrm{Li}^{1}$ Mohamed Darouach ${ }^{2}$, Shujun $\mathrm{Hu}^{3}$ \\ 1. Interdisciplinary Centre for Security, Reliability and Trust, University of Luxembourg, Luxembourg \\ E-mail: lin.pan@uni.lu; Holger.Voos@uni.lu; yumei.li@uni.lu \\ 2. Research Center for Automatic Control of Nancy (CRAN UMR, 7039, CNRS), University of Lorraine, France \\ E-mail: mohamed.darouach@univ-lorraine.fr \\ 3. School of Electric \& Electronic Engineering, Wuhan Polytechnic University, Wuhan 430023, P.R. China \\ E-mail: hushj@whpu.edu.cn
}

\begin{abstract}
This paper investigates the Uncertainty Quantification (UQ) of Exponential Synchronization (ES) problems for a new class of Complex Dynamical Networks (CDNs) with hybrid Time-Varying Delay (TVD) and Non-TimeVarying Delay (NTVD) nodes by using coupling Periodically Intermittent Pinning Control (PIPC) which has three switched intervals in every period. Based on Kronecker product rules, Lyapunov Stability Theory (LST), Cumulative Distribution Function (CDF), and PIPC method, the robustness of the control algorithm with respect to the value of the final time is studied. Moreover, we assume a normal distribution for the time and used the Stochastic Collocation (SC) method [1] with different values of nodes and collocation points to quantify the sensitivity. For different numbers of nodes, the results show that the ES errors converge to zero with a high probability. Finally, to verify the effectiveness of our theoretical results, Nearest-Neighbor Network (NNN) and Barabási-Albert Network (BAN) consisting of coupled non-delayed and delay Chen oscillators are studied and to demonstrate that the accuracies of the ES and PIPC are robust to variations of time.
\end{abstract}

Key Words: Exponential Synchronization (ES), Complex Dynamical Networks (CDNs), Time-varying Delay (TVD), Periodically Intermittent Pinning Control (PIPC)

\section{INTRODUCTION}

In the past few years, UQ and stochastic computation of CDNs have received many research efforts and much attention for many researchers. These also devoted the development of efficient numerical methods in this field. UQ requires an additional method to obtain the stochastic properties which leads to an increase in the computational work compared to the deterministic cases. Therefore, studying the efficiency of the UQ method is very important for the CDNs.

Up to now, several methods to quantify the uncertainties can be found in the related literature. A particular focus is on the design of algorithms that are more efficient than the traditional Monte Carlo (MC) method [2]. A commonly used more efficient method is the Galerkin Polynomial Chaos method [3]. A previous study showed that the SC method only leads to exponential convergence for uniformly distributed uncertain parameters. Recently, J. A. Witteveen and G. Iaccarino have studied subcell resolution in simplex stochastic collocation for spatial discontinuities [4].

Furthermore, most of the current research is primarily concerned with asymptotical or exponential synchronization (ES) of networks via intermittent control $[5,6]$. Because

This work is supported by the National Research Fund, Luxembourg, and cofunded under the Marie Curie Actions of the European Commission (FP7-COFUND). large-scale CDNs with a high order are very complex, it is often the case that only partial state information of key nodes is available in the network outputs and the estimation of the key states of such CDNs becomes necessary. There is a high degree of randomness and unpredictability of chaotic or even super chaotic systems and CDNs with hybrid TVD and NTVD. However, the synchronization of CDNs with hybrid TVD and NTVD will be a key technology e.g. for secure communication. Unfortunately, there are only a few theoretical results and research works dealing with the control and ES for CDNs with hybrid TVD and NTVD nodes by using coupling PIPC, which are important in practical applications.

Inspired by the above mentioned works and discussion$\mathrm{s}$, this paper investigates UQ of ES problems for a new class of CDNs with hybrid TVD and NTVD nodes by using coupling PIPC which has three switched intervals in every period. First, two different PIPC controllers are designed to synchronize the addressed new class of CDN and some useful PIPC criteria are obtained. Herein, PIPC with three switched intervals in every period is used to handle the synchronization problem in CDNs with hybrid TVD and NTVD. Subsequently, by applying LST and Kronecker product rules to the PIPC method, sufficient conditions for ES of the CDNs are proposed and related criteria for the PIPC are derived in some detail. Besides, compared to a control method with one or two intervals, the new results are more flexible, less conservative and its application s- 
cope is broader. This can be verified by some simulation examples comprising a Barabási-Albert Network (BAN) [7] and a Nearest-Neighbor Network (NNN) consisting of coupled non-delayed and delay Chen oscillators.

The rest of the paper is organized as follows : a new class of CDN model is introduced followed by some definition$\mathrm{s}$, assumptions and lemmas in section 2. The PIPC and control criteria for a class of new $\mathrm{CDN}$ are obtained and discussed in section 3. Numerical simulations for UQ and PIPC analysis are presented in section 4. The conclusion of the paper is summarized in section 5 .

\section{Preliminaries and Model Description}

In this section, a novel CDN with hybrid TVD consisting of $N$ coupled nodes is considered. This CDN can be described in the following form:

$$
\dot{x}_{i}(t)=\tilde{\Phi}_{x},(i=1,2, \cdots, N) .
$$

where $\tilde{\Phi}_{x}=C^{(1)} x_{i}(t)+C^{(2)} x_{i}(t-\tau(t))+D^{(1)} f\left(x_{i}(t)\right)+$ $D^{(2)} g\left(x_{i}(t)\right)+B^{(1)} f\left(x_{i}(t-\tau(t))\right)+B^{(2)} g\left(x_{i}(t-\tau(t))\right)+$ $\lambda^{(1)} \sum_{j=1}^{N} G_{i j}^{(1)} \Gamma_{1} x_{j}(t)+\lambda^{(2)} \sum_{j=1}^{N} G_{i j}^{(2)} \Gamma_{2} x_{j}(t-\tau(t))$, $x_{i}(t)=\left(x_{i 1}, x_{i 2}, \cdots, x_{i n}\right)^{T} \in R^{n}$ and $x_{j}(t)=$ $\left(x_{j 1}, x_{j 2}, \cdots, x_{j n}\right)^{T} \in R^{n}$ are the state vectors of node $i$ and $j$, respectively; $\tau(t)$ is an interval TVD; $f$ and $g$ : $R^{n} \rightarrow R^{n}$ are continuously vector-valued functions, and $\lambda^{(1)}$ and $\lambda^{(2)}$ are the strengths of the NTVD and TVD couplings, $G_{i j}^{(1)}=\left(g_{i j}^{(1)}\right)_{N \times N}$ and $G_{i j}^{(2)}=\left(g_{i j}^{(2)}\right)_{N \times N}$ mean the NTVD and TVD weight outer-coupling matrices, respectively, which represent the topological structure and the coupling strength of the complex network. Herein, $g_{i j}^{(1)}$ and $g_{i j}^{(2)}$ are defined as follows: In the case of an existing NTVD coupling connection from node $i$ to node $j(j \neq i)$, then $g_{i j}^{(1)}>0$; otherwise, $g_{i j}^{(1)}=0$; In the case of an existing TVD coupling connection from node $i$ to node $j(j \neq i)$, then $g_{i j}^{(2)}>0$; otherwise, $g_{i j}^{(2)}=0$. $\left(\Gamma_{1}, \Gamma_{2}\right) \subset R^{n \times n}$ are the inner-coupling matrices, which can be described as:

$$
\begin{aligned}
& \Gamma_{1}=\operatorname{diag}\left(\rho_{1}, \rho_{2}, \cdots, \rho_{n}\right), \rho_{i}>0, \\
& \Gamma_{2}=\operatorname{diag}\left(\varrho_{1}, \varrho_{2}, \cdots, \varrho_{n}\right), \varrho_{i}>0 .
\end{aligned}
$$

In order to observe the synchronization behavior of CDN (1), we introduce another CDN which is the response system of the drive system (1). The response system of CDN (1) can be written as

$$
\dot{y}_{i}(t)=\tilde{\Phi}_{y}+u_{i}(t),(i=1,2, \cdots, N) .
$$

where $\tilde{\Phi}_{y}=C^{(1)} y_{i}(t)+C^{(2)} y_{i}(t-\tau(t))+D^{(1)} f\left(y_{i}(t)\right)+$ $D^{(2)} g\left(y_{i}(t)\right)+B^{(1)} f\left(y_{i}(t-\tau(t))\right)+B^{(2)} g\left(y_{i}(t-\tau(t))\right)+$ $\lambda^{(1)} \sum_{j=1}^{N} G_{i j}^{(1)} \Gamma_{1} y_{j}(t)+\lambda^{(2)} \sum_{j=1}^{N} G_{i j}^{(2)} \Gamma_{2} y_{j}(t-\tau(t))$, $y_{i}(t)=\left(y_{i 1}, y_{i 2}, \cdots, y_{i n}\right)^{T} \in R^{n}$ and $y_{j}(t)=$ $\left(y_{j 1}, y_{j 2}, \cdots, y_{j n}\right)^{T} \in R^{n}$ are the state vectors of $\mathrm{n}-$ ode $i$ and $j$, respectively; $u_{i}(t)$ is the appropriate control input that will be designed in the next part to obtain a certain control objective. Let us define the synchronization error vector $e(t)=\left(e_{1}(t), e_{2}(t), \cdots, e_{N}(t)\right)^{T}$ with $e_{i}(t)=x_{i}(t)-y_{i}(t)$ for $i=1,2, \cdots, N$.

First, to prove the results of the next section, we need the following assumptions and lemmas.
Assumption 1. There exist the constants $\varpi_{1} \geq 0$ and $\varpi_{2} \geq 0$, and the positive-definite matrices $\Gamma^{(1)}=\operatorname{diag}\left(\gamma_{1}^{(1)}, \gamma_{2}^{(1)}, \cdots, \gamma_{n}^{(1)}\right)$, and $\Gamma^{(2)}=\operatorname{diag}\left(\gamma_{1}^{(2)}, \gamma_{2}^{(2)}, \cdots, \gamma_{n}^{(2)}\right)$ such that functions $f$ and $g$ satisfying the following inequalities:

$$
\begin{aligned}
& {\left[x_{i}(t)-y_{i}(t)\right]^{T}\left[f\left(x_{i}(t)\right)-f\left(y_{i}(t)\right)\right] } \\
\leq & \varpi_{1}\left[x_{i}(t)-y_{i}(t)\right]^{T} \Gamma^{(1)}\left[x_{i}(t)-y_{i}(t)\right], \\
& {\left[x_{i}(t)-y_{i}(t)\right]^{T}\left[g\left(x_{i}(t)\right)-g\left(y_{i}(t)\right)\right] } \\
\leq & \varpi_{2}\left[x_{i}(t)-y_{i}(t)\right]^{T} \Gamma^{(2)}\left[x_{i}(t)-y_{i}(t)\right] .
\end{aligned}
$$

where $x_{i}(t), y_{i}(t) \in R^{n}$.

Assumption 2. For the vector-valued function$s \quad f(t, x(t), x(t-\tau(t)))$ and $g(t, x(t), x(t-\tau(t)))$, suppose the uniform semi-Lipschitz condition with respect to the time $t$ holds, i.e., for any $x(t), y(t), x(t-\tau(t)), y(t-\tau(t)) \in R^{n}$, there exist positive constants $L_{1}^{(1)}>0, L_{1}^{(2)}>0, L_{2}^{(1)}>0$ and $L_{2}^{(2)}>0$ such that:

$$
\begin{aligned}
& {[x(t-\tau(t))-y(t-\tau(t))]^{T}[g(t, x(t), x(t-\tau(t)))} \\
& -g(t, y(t), y(t-\tau(t)))] \\
& \leqslant L_{1}^{(2)}[x(t)-y(t)]^{T}[x(t)-y(t)] \\
& \quad+L_{2}^{(2)}[x(t-\tau(t)) \\
& -y(t-\tau(t))]^{T}[x(t-\tau(t))-y(t-\tau(t))] .
\end{aligned}
$$

Lemma 1. There exits a class of continuous functions $v$ : $\left[t_{0}-\tau, \infty\right) \rightarrow[0, \infty)$ satisfying $[8,9]:$

$$
\dot{v}(t) \leq-a v(t)+b \sup _{t-\tau \leq s \leq t} v(s)
$$

for $t \geq t_{0}$. If $0<b<a$, then

$$
v(t) \leq \sup _{t_{0}-\tau \leq \varphi \leq t_{0}} v(\varphi) \exp \left\{-\lambda\left(t-t_{0}\right)\right\}, \quad t \geq t_{0},
$$

where $0<\lambda$ is the positive unique solution of the equation $-a+\lambda+b \exp (\lambda \tau)=0$.

Lemma 2. There exits a class of continuous functions $v$ : $\left[t_{0}-\tau, \infty\right) \rightarrow[0, \infty)$ satisfying $[8,9]:$

$$
\dot{v}(t) \leq \zeta_{1} v(t)+\zeta_{2} \sup _{t-\tau \leq s \leq t} v(s)
$$

for $t \geq t_{0}$. If $0<\zeta_{1}, 0<\zeta_{2}$, then

$$
v(t) \leq \sup _{t_{0}-\tau \leq \varphi \leq t_{0}} v(\varphi) \exp \left\{\left(\zeta_{1}+\zeta_{2}\right)\left(t-t_{0}\right)\right\}, \quad t \geq t_{0} .
$$

In this work, the PIPC scheme with its three switched intervals are proposed for the first time and depicted in Fig. 1, in which the red, yellow and blue intervals are control durations or control widths and the white interval is non-control duration or non-control width, respectively.

As we see in Fig. 1, $T_{1}, T_{2}$ and $T_{3}$ are three intervals that alternately appear in every period. If $T_{1}>0, T_{2}>0, T_{3}>$ $0, T=T_{1}+T_{2}+T_{3}, \delta_{1}=c d_{1}, \delta_{2}=c d_{2}$ and $\delta_{3}=c d_{3} . \delta_{1}$, 


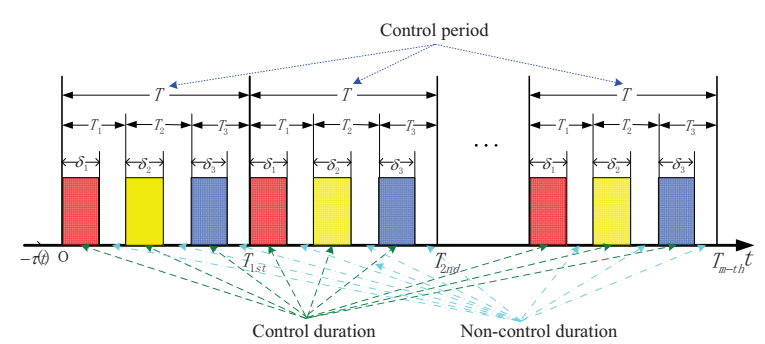

Fig. 1: The portraits of the PIPC scheme with its three switched intervals.

$\delta_{2}$ and $\delta_{3}$ are called the control durations or control widths in $T_{1}, T_{2}$ and $T_{3}$, respectively. $\theta(1>\theta>0)$ is the rate of control duration or control width in the control periods, and it satisfies $\theta=\frac{c d_{1}}{T_{1}}=\frac{c d_{2}}{T_{2}}=\frac{c d_{3}}{T_{3}}=\frac{c d_{1}+c d_{2}+c d_{3}}{T}$. $(1-\theta) T_{1},(1-\theta) T_{2},(1-\theta) T_{3}$ are called non-control durations or non-control widths in control intervals $T_{1}, T_{2}$ and $T_{3}$, respectively.

The PIPC using three switched periods are clarified in detail as follows. Some intermittent controllers are added to partial nodes of network (1). Noting that $\Omega_{11}^{m}=$ $\left[m T, m T+\theta T_{1}\right)$ is the control width in interval $T_{1}, \Omega_{12}^{m}=$ $\left[m T+\theta T_{1}, m T+T_{1}\right)$ is the non-control width in interval $T_{1}, \Omega_{21}^{m}=\left[m T+T_{1}, m T+T_{1}+\theta T_{2}\right)$ is the control width in interval $T_{2}, \Omega_{22}^{m}=\left[m T+T_{1}+\theta T_{2}, m T+T_{1}+T_{2}\right)$ is the non-control width in interval $T_{2}, \Omega_{31}^{m}=\left[m T+T_{1}+\right.$ $\left.T_{2}, m T+T_{1}+T_{2}+\theta T_{3}\right)$ is the control width in interval $T_{3}, \Omega_{32}^{m}=\left[m T+T_{1}+T_{2}+\theta T_{3}, m T+T_{1}+T_{2}+T_{3}\right)$ is the non-control width in interval $T_{3}$, It is obvious that their respective intervals with TVD are $\bar{\Omega}_{11}^{m}=[m T-\tau(t), m T+$ $\left.\theta T_{1}-\tau(t)\right), \bar{\Omega}_{12}^{m}=\left[m T+\theta T_{1}-\tau(t), m T+T_{1}\right)-\tau(t)$, $\bar{\Omega}_{21}^{m}=\left[m T+T_{1}-\tau(t), m T+T_{1}+\theta T_{2}-\tau(t)\right)$, $\bar{\Omega}_{22}^{m}=\left[m T+T_{1}+\theta T_{2}-\tau(t), m T+T_{1}+T_{2}-\tau(t)\right)$, $\bar{\Omega}_{31}^{m}=\left[m T+T_{1}+T_{2}-\tau(t), m T+T_{1}+T_{2}+\theta T_{3}-\tau(t)\right)$, and $\bar{\Omega}_{32}^{m}=\left[m T+T_{1}+T_{2}+\theta T_{3}-\tau(t), m T+T_{1}+T_{2}+\right.$ $\left.T_{3}-\tau(t)\right)$, where $m \in M^{+}=\{0,1,2, \cdots\}$.

Without loss of generality, suppose that the first $l(1 \leq l<$ $N)$ nodes are selected and pinned, leading to the following controlled dynamical network:

$$
\left\{\begin{aligned}
\dot{x}_{i}(t)= & \tilde{\Phi}_{x}+d_{i}(t)\left(x_{i}(t)-s(t)\right) \\
& +d_{i}(t-\tau(t))\left(x_{i}(t-\tau(t))-s(t-\tau(t))\right) \\
& 1 \leq i \leq l \\
\dot{y}_{i}(t)= & \tilde{\Phi}_{y}, l+1 \leq i \leq N
\end{aligned}\right.
$$

where $d_{i}(t)>0$ and $d_{i}(t-\tau(t))>0$ are the intermittent feedback control gains defined as follows:

$$
\begin{gathered}
d_{i}(t)=\left\{\begin{array}{l}
-d_{i}, t \in \Omega_{11}^{m} \cup \Omega_{21}^{m} \cup \Omega_{31}^{m}, 1 \leq i \leq l, \\
0, t \in \Omega_{12}^{m} \cup \Omega_{22}^{m} \cup \Omega_{32}^{m}, 1 \leq i \leq N .
\end{array}\right. \\
d_{i}(t-\tau(t))=\left\{\begin{array}{l}
-d_{i}, t \in \bar{\Omega}_{11}^{m} \cup \bar{\Omega}_{21}^{m} \cup \bar{\Omega}_{31}^{m}, 1 \leq i \leq l, \\
0, t \in \bar{\Omega}_{12}^{m} \cup \bar{\Omega}_{22}^{m} \cup \bar{\Omega}_{32}^{m}, 1 \leq i \leq N .
\end{array}\right.
\end{gathered}
$$

where $T>0$ is the control period, $\delta>0$ is called the control duration (control width), and $m=0,1,2, \cdots$.
Let the first $l$ nodes be selected and pinned, and the controllers $u_{i}$ can be described by

$$
u_{i}=\left\{\begin{array}{c}
-d_{i}\left[e_{i}(t)+e_{i}(t-\tau(t))\right], \\
t \in \Omega_{11}^{m} \cup \Omega_{21}^{m} \cup \Omega_{31}^{m}, 1 \leq i \leq l, \\
0, t \in \Omega_{11}^{m} \cup \Omega_{21}^{m} \cup \Omega_{31}^{m}, l+1 \leq i \leq N \\
0, t \in \Omega_{12}^{m} \cup \Omega_{22}^{m} \cup \Omega_{32}^{m}, 1 \leq i \leq N .
\end{array}\right.
$$

Let $e_{i}(t)=x_{i}(t)-s(t)(1 \leq i \leq N)$ and $e_{i}(t-\tau(t))=$ $x_{i}(t-\tau(t))-s(t-\tau(t))(1 \leq i \leq N)$ be synchronization errors, and $\theta=\frac{\delta}{T}$ be the ratio of the control duration $\delta$ to the control period $T$ called control rate. According to the control laws (12), then the dynamical error system can be derived as:

$$
\left\{\begin{array}{l}
\dot{e}_{i}(t)=\tilde{\Phi}_{e}-d_{i}\left[e_{i}(t)+e_{i}(t-\tau(t))\right], \\
t \in \Omega_{11}^{m} \cup \Omega_{21}^{m} \cup \Omega_{31}^{m}, 1 \leq i \leq l ; \\
\dot{e}_{i}(t)=\tilde{\Phi}_{e}, \\
t \in \Omega_{11}^{m} \cup \Omega_{21}^{m} \cup \Omega_{31}^{m}, l+1 \leq i \leq N ; \\
\dot{e}_{i}(t)=\tilde{\Phi}_{e}, \\
t \in \Omega_{12}^{m} \cup \Omega_{22}^{m} \cup \Omega_{32}^{m}, 1 \leq i \leq N .
\end{array}\right.
$$

Where $\tilde{\Phi}_{e}=C^{(1)} e_{i}(t)+C^{(2)} e_{i}(t-\tau(t))+$ $D^{(1)} \tilde{f}\left(x_{i}(t)\right)+D^{(2)} \tilde{g}\left(x_{i}(t)\right)+B^{(1)} \tilde{f}\left(x_{i}(t-\tau(t))\right)+$ $B^{(2)} \tilde{g}\left(x_{i}(t-\tau(t))\right)+\lambda^{(1)} \sum_{j=1}^{N} G_{i j}^{(1)} \Gamma^{(1)} e_{j}(t)+$ $\lambda^{(2)} \sum_{j=1}^{N} G_{i j}^{(2)} \Gamma^{(2)} e_{j}(t \quad-\tau(t)), \quad \tilde{f}\left(x_{i}(t)\right)=$ $f\left(x_{i}(t)\right)-f(s(t)), \tilde{g}\left(x_{i}(t)\right)=g\left(x_{i}(t)\right)-g(s(t))$; $\tilde{f}\left(x_{i}(t-\tau(t))\right)=f\left(x_{i}(t-\tau(t))\right)-f(s(t-\tau(t))), \tilde{g}\left(x_{i}(t-\right.$ $\tau(t)))=g\left(x_{i}(t-\tau(t))\right)-g(s(t-\tau(t)))$. It is easy to achieve global ES of the controlled dynamical network (9) when the zero solutions of the error system (13) are globally exponentially stable.

\section{PIPC and control criteria for a class of new CDNs}

Based on the PIPC of the network (1), the main results regarding ES criteria are derived in this section. The key results are achieved using the Lyapunov functional method and Kronecker product rules and a suitable controller is designed to realize the PIPC of network (1).

The following theorem gives the main results for the synchronization and control of the novel CDN. First of all, we assume that $\left\|\Gamma_{1}\right\|=\rho^{(1)}>0$, and $\left\|\Gamma_{2}\right\|=\rho^{(2)}>0$, and denote $\rho_{\min }^{(1)}$ and $\rho_{\min }^{(2)}$ as the minimum eigenvalues of matrices $\left(\Gamma_{1}+\Gamma_{1}^{T}\right) / 2$ and $\left(\Gamma_{2}+\Gamma_{2}^{T}\right) / 2$, respectively. Let $\rho_{\min }^{(1)} \neq 0$ and $\rho_{\min }^{(2)} \neq 0$. Suppose that the conditions $\hat{G}^{(1) s}=\left(\hat{G}^{(1)}+\hat{G}^{(1) T}\right) / 2$ and $\hat{G}^{(2) s}=\left(\hat{G}^{(2)}+\hat{G}^{(2) T}\right) / 2$ hold, where $\hat{G}^{(1)}$ is a modified matrix of $G^{(1)}$ where the diagonal elements $g_{i i}^{(1)}$ are replaced by $\left(\rho_{\min }^{(1)} / \rho^{(1)}\right) g_{i i}^{(1)}$, and $\hat{G}^{(2)}$ is a modified matrix of $G^{(2)}$ where the diagonal elements $g_{i i}^{(2)}$ are replaced by $\left(\rho_{\min }^{(2)} / \rho^{(2)}\right) g_{i i}^{(2)}$. Then $\hat{G}^{(1)}$ and $\hat{G}^{(2)}$ are irreducible symmetric matrices with offdiagonal nonnegative elements therein. The main results are obtained as follows:

Theorem 1. Assuming that assumptions 1 and 2 hold, let $\delta=\xi_{1} T<T-\tau\left(0<\xi_{1}\right)$ and $\tau=\xi_{2} T<\delta\left(0<\xi_{2}\right)$. If 
there exist positive constants $a_{1}, a_{2}$, and $d_{i}, i=1, \cdots, l$, such that

(a) $\left(C^{(1)}+C^{(2)}+2 D^{(1)} L_{1}^{(1)}+2 D^{(2)} L_{1}^{(2)}+2 B^{(1)} L_{1}^{(3)}+\right.$ $\left.2 B^{(2)} L_{1}^{(4)}+a_{1}\right) I_{N}$ $+\lambda^{(1)} \rho^{(1)} \hat{G}^{(1) s}+\lambda^{(2)} \rho^{(2)} \hat{G}^{(2) s}-2 D<0 ;$

(b) $\left(C^{(1)}+C^{(2)}+2 D^{(1)} L_{1}^{(1)}+2 D^{(2)} L_{1}^{(2)}+2 B^{(1)} L_{1}^{(3)}+\right.$ $\left.2 B^{(2)} L_{1}^{(4)}\right) I_{N}$ $+\lambda^{(1)} \rho^{(1)} \hat{G}^{(1) s}+\lambda^{(2)} \rho^{(2)} \hat{G}^{(2) s}-\left(a_{2}-a_{1}\right) I_{N}<0$;

(c) $-a_{1}+2\left(D^{(1)} L_{2}^{(1)}+D^{(2)} L_{2}^{(2)}+B^{(1)} L_{2}^{(3)}+B^{(2)} L_{2}^{(4)}\right)<$ 0

(d) $\left(a_{2}-a_{1}\right)+2\left(D^{(1)} L_{2}^{(1)}+D^{(2)} L_{2}^{(2)}+B^{(1)} L_{2}^{(3)}+\right.$ $\left.B^{(2)} L_{2}^{(4)}\right)<0$;

(e) $-\left(\zeta_{1}+\zeta_{2}\right)\left(1-\xi_{1}\right)+\lambda\left(\xi_{1}-\xi_{2}\right)>0$.

where $D=\operatorname{diag}(d_{1}, \cdots, d_{l}, \underbrace{0, \cdots, 0}_{N-l})$, and $\lambda>0$ is the positive unique solution of the equation $-2 a_{1} V(t)+\lambda+$ $4\left(D^{(1)} L_{2}^{(1)}+D^{(2)} L_{2}^{(2)}+B^{(1)} L_{2}^{(3)}+B^{(2)} L_{2}^{(4)}\right) \exp \{\lambda \tau\}=$ 0 . Then the controlled dynamical network (9) can achieve ES under the control of the PIPC controllers (12) with appropriate control time.

Proof. Let $e(t)=\left(\left\|e_{1}(t)\right\|,\left\|e_{2}(t)\right\|, \cdots,\left\|e_{N}(t)\right\|\right)^{T}$, and consider the following Lyapunov function:

$$
V(t)=\frac{1}{2} e^{T}(t) e(t)=\frac{1}{2} \sum_{i=1}^{N} e_{i}^{T}(t) e_{i}(t)
$$

Using assumptions (1) and (2), conditions $(a)$ and $(b)$, the derivative of $V(t)$ with respect to time $t$ along the solutions of equation (13) can be then calculated and get the results. Namely, we have

$$
\begin{gathered}
\dot{V}(t) \leq- \\
+a_{1} V(t)+4\left(D^{(1)} L_{2}^{(1)}+D^{(2)} L_{2}^{(2)}\right. \\
\left.+B^{(1)} L_{2}^{(3)}+B^{(2)} L_{2}^{(4)}\right) V(t-\tau(t)) \\
t \in \Omega_{11}^{m} \cup \Omega_{21}^{m} \cup \Omega_{31}^{m}, m \in M^{+} \\
\dot{V}(t) \leq 2\left(a_{2}-a_{1}\right) V(t)+4\left(D^{(1)} L_{2}^{(1)}+D^{(2)} L_{2}^{(2)}\right. \\
\left.+B^{(1)} L_{2}^{(3)}+B^{(2)} L_{2}^{(4)}\right) V(t-\tau(t)) \\
t \in \Omega_{12}^{m} \cup \Omega_{22}^{m} \cup \Omega_{32}^{m}, m \in M^{+}
\end{gathered}
$$

Within every single period $T$ and also $T_{i}(i=1,2,3)$, one has

$$
\begin{gathered}
V(t) \leq \sup _{T_{i}-\tau \leq \theta \leq T_{i}} V(\theta) \exp \left\{-\lambda\left(t-T_{i}\right)\right\} \\
t \in \Omega_{11}^{m} \cup \Omega_{21}^{m} \cup \Omega_{31}^{m}, m \in M^{+} \\
V(t) \leq \sup _{T_{i}+\delta-\tau \leq \theta \leq T_{i}+\delta} V(\theta) \exp \left\{\left(\zeta_{1}+\zeta_{2}\right)\left(t-T_{i}-\delta\right)\right\} \\
t \in \Omega_{12}^{m} \cup \Omega_{22}^{m} \cup \Omega_{32}^{m}, m \in M^{+}
\end{gathered}
$$

$$
\begin{aligned}
V(t) \leq & \sup _{-\tau \leq \theta \leq 0} V(\theta) \exp \left\{-\left[-\left(\zeta_{1}+\zeta_{2}\right)\left(1-\xi_{1}\right)\right.\right. \\
& \left.\left.+\lambda\left(\xi_{1}-\xi_{2}\right)\right] t+\lambda\left(\xi_{1}-\xi_{2}\right) \delta\right\} \\
& t \in \Omega_{11}^{m} \cup \Omega_{21}^{m} \cup \Omega_{31}^{m}, m \in M^{+} .
\end{aligned}
$$

$$
\begin{aligned}
V(t) \leq & \sup _{-\tau \leq \theta \leq 0} V(\theta) \exp \left\{-\left[-\left(\zeta_{1}+\zeta_{2}\right)\left(1-\xi_{1}\right)\right.\right. \\
& \left.\left.+\lambda\left(\xi_{1}-\xi_{2}\right)\right] t+\lambda\left(\xi_{1}-\xi_{2}\right) \delta\right\}, \\
& t \in \Omega_{12}^{m} \cup \Omega_{22}^{m} \cup \Omega_{32}^{m}, m \in M^{+} .
\end{aligned}
$$

So far, as for any $t \geq 0$, or $t \in \Omega_{11}^{m} \cup \Omega_{21}^{m} \cup \Omega_{31}^{m} \cup \Omega_{12}^{m} \cup$ $\Omega_{22}^{m} \cup \Omega_{32}^{m}, m \in M^{+}$, combining (16), (17) with (18), (19), we can obtain

$$
\begin{aligned}
V(t) & \leq \sup _{-\tau \leq \theta \leq 0} V(\theta) \exp \left\{-\left[-\left(\zeta_{1}+\zeta_{2}\right)\left(1-\xi_{1}\right)\right.\right. \\
& \left.\left.+\lambda\left(\xi_{1}-\xi_{2}\right)\right] t+\lambda\left(\xi_{1}-\xi_{2}\right) \delta\right\} .
\end{aligned}
$$

Based on equation (14), we then get

$$
\begin{aligned}
& \|e(t)\|_{2} \leq[2 V(t)]^{1 / 2} \\
\leq & {\left[2 \sup _{-\tau \leq \theta \leq 0} V(\theta)\right]^{1 / 2} } \\
& \exp \left\{-\frac{\left[-\left(\zeta_{1}+\zeta_{2}\right)\left(1-\xi_{1}\right)+\lambda\left(\xi_{1}-\xi_{2}\right)\right]}{2} t\right. \\
& \left.+\frac{\lambda\left(\xi_{1}-\xi_{2}\right) \delta}{2}\right\} .
\end{aligned}
$$

Due to the condition (e): $-\left(\zeta_{1}+\zeta_{2}\right)\left(1-\xi_{1}\right)+\lambda\left(\xi_{1}-\right.$ $\left.\xi_{2}\right)>0$, we can get the complete conclusion for Theorem 1. For Assumptions (1)-(2) and conditions (a-e), we have $V(t)<0$. Based on the LST, the results can be derived for $\lim _{t \rightarrow \infty}\left\|e_{i}(t)\right\|_{2}=0$ and $\lim _{t \rightarrow \infty}\left\|e_{i}(t-\tau(t))\right\|_{2}=0$. Accord$t \rightarrow \infty$
ingly, the error system $(13)$ is asymptotically stable which shows the stability of ES with PIPC of the system 9.

In the next section, some simulations and figures are presented to illustrate the results of Theorem 1.

\section{Some numerical simulations for PIPC and UQ analysis}

In this section, some numerical simulations for PIPC and UQ analysis are given to verify the effectiveness of the proposed ES schemes and PIPC criteria for synchronizing a $\mathrm{CDN}$ with time-delayed dynamical nodes onto a chaotic trajectory and a periodic orbit. Usually, chaotic attractors will be seen as nodes or oscillators of BANs and NNNs by using PIPC. A BAN with 130 nodes is selected as the nondelay coupling network with the random pinning strategy, see Fig 2(a). The BAN is constructed using the BarabásiAlbert Model (BAM) [7] with $m_{0}=3$ starting nodes. Furthermore, a NNN with 130 nodes is selected as the coupling delay $\tau(t)$ network, whose average value of nodes degree is 6 , see Fig 2(b).

In these dynamical systems, each node is a Chen system [10], which satisfies assumptions 1 and 2, and the $i$ th 


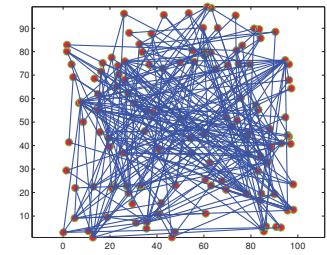

(a) BAN.

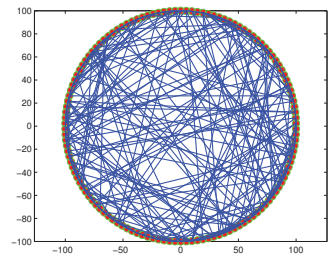

(b) NNN.
Fig. 2: The schematic illustration of BAN and NNN consisting of 130 nodes.

node can be written as follows:

$$
\left\{\begin{array}{l}
\dot{w}_{i 1}=a\left(w_{i 2}-w_{i 1}\right) \\
\dot{w}_{i 2}=(c-a) w_{i 1}-w_{i 1} w_{i 3}+c w_{i 2}, \quad 1 \leq i \leq 130, \\
\dot{w}_{i 3}=w_{i 1} w_{i 2}-b w_{i 3} .
\end{array}\right.
$$

Here, we set $a=35 ; b=3 ; c=28$. The system shows chaotic behavior, see [10].

We consider a CDN (1) consisting of 130 identical Chen chaotic system as time-delayed nodes for simplicity, where $\Gamma_{1}=\operatorname{diag}(1.01,1.05,1.03), \Gamma_{2}=\operatorname{diag}(1.02,1.06,1.04)$, $G_{i j}^{(1)}=\left(g_{i j}^{(1)}\right)_{130 \times 130}$, and $G_{i j}^{(2)}=\left(g_{i j}^{(2)}\right)_{130 \times 130}$ is a symmetrically diffusive coupling matrix with $g_{i j}^{(2)}=1$ or $0(i \neq$ $j$ ), and the coupling strength $c=40$. In addition, let $\tau(t)=0.010, \delta=0.031, \xi_{1}=2$, and $\xi_{2}=3$, i.e. for all $i=1,2, \cdots, l(l=30)$ holds. By taking the control rate $\theta=\frac{\delta_{1}+\delta_{2}+\delta_{3}}{T}=\frac{\delta}{T}=\frac{0.031}{0.100}=0.3100$, and choosing the control period $T=0.100$, the sufficient conditions of Theorem 1 are satisfied.

The initial conditions of the numerical simulations are as follows: $x_{i}(0)=(-10+0.5 i, 18+0.5 i, 20+0.5 i)^{T}$, $s(0)=(-10,18,20)^{T}$, where $1 \leq i \leq 130$, and $d_{1}(0)=$ $\cdots=d_{30}(0)=1$. The synchronous errors $e_{i}(t)$ are illustrated in Figs. 3(a)-3(d) and the values for the control gains after synchronization satisfy $d_{i} \leq d_{30}=1,1 \leq i \leq 30$, which illustrate that the adaptive control approach can obtain a more applicable control gain:

$$
d_{i}(t)=\left\{\begin{array}{c}
-d_{i}, \dot{d}_{i}=q_{i} e_{i}^{T}(t) e_{i}(t), d_{i}(0)=0, q_{i}>0 \\
\quad m T \leq t \leq m T+\delta, 1 \leq i \leq 30 \\
0, \quad m T \leq t \leq m T+\delta, 31 \leq i \leq 130 \\
0, \quad m T+\delta \leq t \leq(m+1) T+\delta \\
1 \leq i \leq 130
\end{array}\right.
$$

Clearly, the controlled network (9) is globally exponentially stable at zero under the intermittent controllers with $l=30$ and the states of the controlled network approach to the desired orbits with PIPC and TVD as shown in Figs. 4(a)-4(d). The desired orbits with initial conditions $x_{1}(0)=-10, x_{2}(0)=18, x_{3}(0)=20$ are shown in Fig. 4.

Furthermore, we calculate the corresponding Time(s) and $u_{k}=e_{1}(1, T)$ for different $n$ using MATLAB. With the results given in Table 1, we study the robustness of the control algorithm with respect to the value of the final time $T$. To that end, we assumed a normal distribution for $T$ and

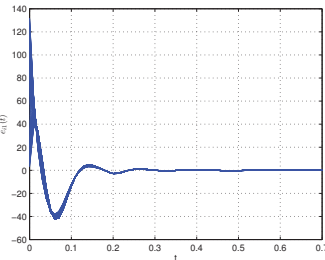

(a) The ES of $e_{i 1}(t)$.

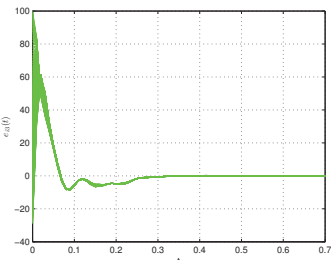

(c) The ES of $e_{i 3}(t)$.

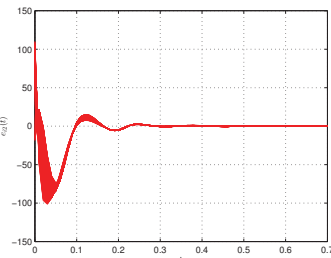

(b) The ES of $e_{i 2}(t)$.

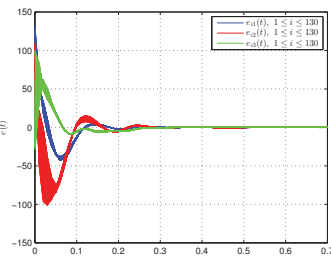

(d) Comparing the three states errors.
Fig. 3: Temporal responses of the ES $e_{i j}(t)(1 \leq i \leq$ $130,1 \leq j \leq 3)$ for the controlled CDN with TVD.
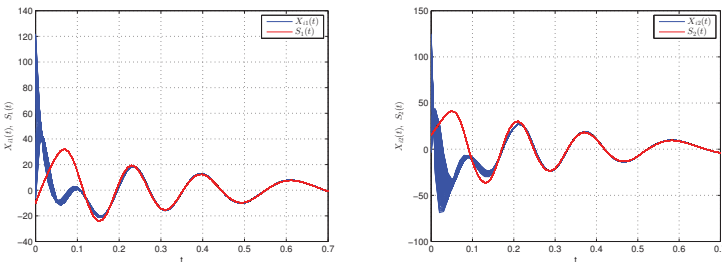

(a) Desired orbit $S_{1}(t)$ and the (b) Desired orbit $S_{2}(t)$ and the $X_{i 1}(t)$.

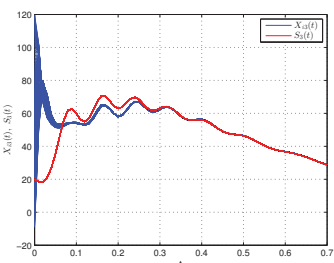
$X_{i 2}(t)$.

(c) Desired orbit $S_{3}(t)$ and the (d) Comparing the three states er$X_{i 3}(t)$. rors.

Fig. 4: Temporal responses of the desired orbit $S_{j}(t)$ and the $X_{i j}(t)(1 \leq i \leq 130,1 \leq j \leq 3)$ for the controlled CDN with TVD.

we used the SC method [1] with $n=3$, and it's collocation points $T_{k}$ to quantify the sensitivity in terms of the CDF. If we adjust the evolutionary time of ES with PIPC, and set time as $t=2.2679 \mathrm{~s}, t=4.0000 \mathrm{~s}$, and $t=5.7321 \mathrm{~s}$ in the following Table. 1, then the ES error of the simulation results are shown in Figs. 5(a), 5(c), and 5(e). It can be clearly seen tat the errors converge to zero in the Figures. The temporal responses of the desired orbit $S_{1}(t)$ and the $X_{i 1}^{(k)}(t)$ in the CDNs with PIPC for $k=1, \cdots, 3$ are shown in Figs. 5. Moreover, the UQ analysis and distribution for $e_{(1, T)}$ and the ES points for $n=3$ are shown in Figs. 6.

The Fig. 5 shows the ES $e_{1}$ for the different collocation points $T_{k}$ for $n=3$ for all 130 nodes with PIPC in the 
Table 1: The variables and values for UQ of ES with PIPC when $n=3(k=1, \cdots, n)$ and mean $_{t}=4$.

\begin{tabular}{|c|c|c|c|}
\hline $\begin{array}{ll}\text { Variables } & k\end{array}$ & $k=1$ & $k=2$ & $k=3$ \\
\hline Time(s) & 2.267949192 & 4.000 & 5.7320508076 \\
\hline$u_{k}=e_{1}(1, T)$ & -0.0105 & -0.2179 & -0.1427 \\
\hline
\end{tabular}

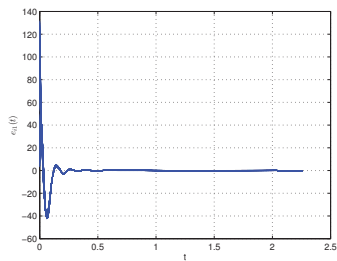

(a) $k=1$.

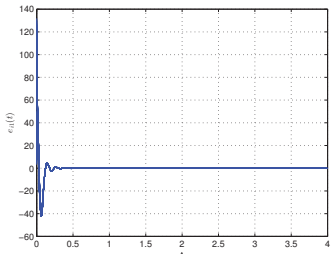

(c) $k=2$

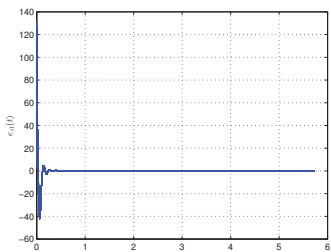

(e) $k=3$

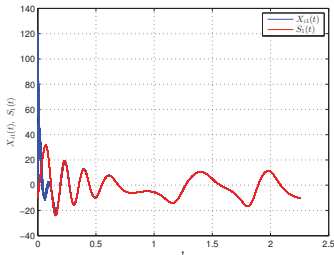

(b) $k=1$.

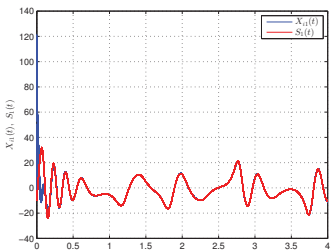

(d) $k=2$

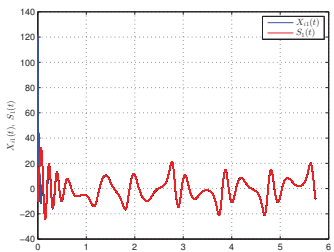

(f) $k=3$
Fig. 5: Simulated portraits of the ES error $e_{i 1}$, desired orbit $S_{1}(t)$ and the $X_{i 1}^{(k)}(t)$ in the CDNs with PIPC for $k=$ $1, \cdots, 3$.

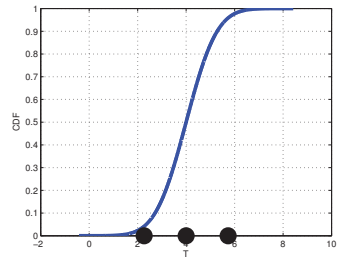

(a) $C D F$ and $T$ for $n=3$.

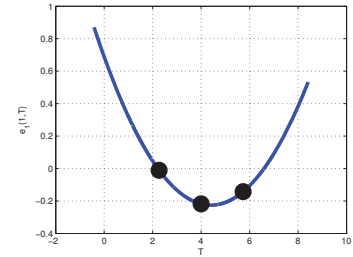

(c) $e_{(1, T)}$ and $T$ for $n=3$.

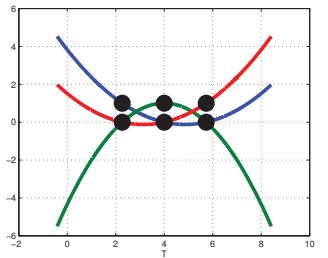

(b) $L$ and $T$ for $n=3$.

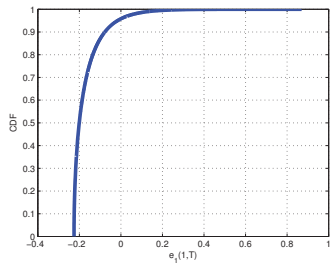

(d) $C D F$ and $e_{(1, T)}$ for $n=3$.
Fig. 6: UQ analysis and distribution for $e_{(1, T)}$ and the ES points for $n=3$. system. In addition, this figure also shows the temporal responses of the desired orbit $S_{1}(t)$ and the $X_{i 1}^{(k)}(t)$ in the CDNs with PIPC for $k=1, \cdots, 3$. The Fig. 6 shows the resulting distribution of $e_{1}(1, T)$. The $\mathrm{ES} e_{1}(1, T)$ are close to zero with a high probability. These results confirm the applicability of the proposed method.

\section{Conclusions}

In this paper, we investigated the UQ of ES problems for a new class of CDNs with hybrid TVD and NTVD nodes by using coupling PIPC with three switched intervals in every period. Based on the LST, Kronecker product rules, CDF and PIPC method, the robustness of the control algorithm with respect to the value of the final time has been studied. Moreover, we assumed a normal distribution for time and used the SC method with different values of nodes $n$ and collocation points $T_{k}$ to quantify the sensitivity. The results show that the ES errors converge to zero with a high probability and for different numbers of nodes. Finally, BAN and NNN consisting of coupled non-delayed and delay Chen systems are finally presented as examples to verify the effectiveness of the addressed theoretical results. In the future, we will study in-depth uncertainty quantification for system with distributed time delays stochastic perturbation and hybrid optimal control.

\section{REFERENCES}

[1] G. Loeven, J. Witteveen, H. Bijl, Probabilistic collocation: an efficient non-intrusive approach for arbitrarily distributed parametric uncertainties, in: 45th AIAA Aerospace Sciences Meeting and Exhibit, Reno, Nevada, 2007, p. 317.

[2] J. D. Jakeman, A. Narayan, D. Xiu, Minimal multi-element stochastic collocation for uncertainty quantification of discontinuous functions, Journal of Computational Physics 242 (2013) 790-808.

[3] I. Babuška, F. Nobile, R. Tempone, A stochastic collocation method for elliptic partial differential equations with random input data, SIAM Journal on Numerical Analysis 45 (3) (2007) 1005-1034.

[4] J. A. Witteveen, G. Iaccarino, Subcell resolution in simplex stochastic collocation for spatial discontinuities, Journal of Computational Physics 251 (2013) 17-52.

[5] J. Mei, M. Jiang, X. Wang, J. Han, S. Wang, Finite-time synchronization of drive-response systems via periodically intermittent adaptive control, Journal of the Franklin Institute 351 (5) (2014) 2691-2710.

[6] Y. Liang, X. Wang, Synchronization in complex networks with non-delay and delay couplings via intermittent control with two switched periods, Physica A: Statistical Mechanics and its Applications 395 (2014) 434-444.

[7] R. Albert, A.-L. Barabási, Statistical mechanics of complex networks, Reviews of modern physics 74 (1) (2002) 47.

[8] W. Xia, J. Cao, Pinning synchronization of delayed dynamical networks via periodically intermittent control, Chaos: An Interdisciplinary Journal of Nonlinear Science 19 (1) (2009) 013120

[9] S. Cai, Q. He, J. Hao, Z. Liu, Exponential synchronization of complex networks with nonidentical time-delayed dynamical nodes, Physics Letters A 374 (25) (2010) 2539 2550.

[10] G. Chen, T. Ueta, Yet another chaotic attractor, International Journal of Bifurcation and Chaos 9 (7) (1999) 14651466. 